\title{
Nonprofit Hospital Community Benefit Spending and Readmission Rates
}

\author{
Krisda H. Chaiyachati, MD, MPH, MSHP, ${ }^{1,2}$ Mingyu Qi, MSc, ${ }^{1}$ and Rachel M. Werner, MD, $\mathrm{PhD}^{1-3}$
}

\begin{abstract}
Addressing social determinants of health may help hospitals improve population health and outcomes that are tied to financial incentives, such as readmission rates. Whether this strategy is effective remains unknown. The authors measure the association between nonprofit hospital spending on their community's social needs and Medicare readmission rates. This is a retrospective cohort study (July 2013 to June 2014) that combines Internal Revenue Service tax reports of community benefit spending by private, acute care, nonprofit hospitals with Medicare readmissions data for residents of their surrounding community, the hospital's zip code. Total community benefit spending and 2 of the largest subsets of spending-health care-related and communitydirected spending - were measured and normalized as the proportion of each hospital's total expenditure. Associations between quintiles of community benefit spending and Medicare readmission rates were measured using discharge-level multivariate linear regression, adjusting for patient, hospital, community characteristics, and local public health department spending. The sample consisted of 1405 nonprofit hospitals with 341,913 discharges. Associations between readmission rates and total community benefit spending and the health carerelated subset were not statistically significant. Discharges from hospitals in the upper quintiles of communitydirected spending were associated with lower readmission rates by 0.82 to 1.21 absolute percentage points $(P=0.01$ to $<0.001)$ compared to the lowest quintile. The magnitude of associations between communitydirected spending and readmissions was larger for preventable readmissions and smaller when including beneficiaries beyond the hospital's zip code. These associations suggest that community-directed spending may be associated with better health care outcomes.
\end{abstract}

Keywords: readmissions, social determinants of health, community benefit, nonprofit hospitals, community investments

\section{Introduction}

A MAJOR GOAL OF Medicare's Hospital Readmissions Reduction Program is to hold hospitals accountable for patient outcomes that occur after hospital discharge - hospitals with higher-than-expected 30-day readmission rates face financial penalties. This reform has faced criticism because readmissions can be influenced by social determinants of health (eg, poverty, homelessness, food insecurity), often perceived as beyond the hospital's control. ${ }^{1,2}$ Indeed, hospitals that care for high proportions of socially at-risk patients, such as low-income minorities, are more likely to have higher 30-day readmissions relative to hospitals serving fewer socially at-risk patients. $^{3-5}$ Thus, hospitals are facing increasing pressure to effectively manage social determinants of health in their communities - or the conditions in which people live, work, and age. ${ }^{6}$

Although addressing social determinants of health is increasingly incorporated into population health strategies for specific diseases, these interventions have had inconsistent effects on improving patient outcomes. ${ }^{2,7}$ Moreover, these interventions are most often led by public health departments, not hospitals, who may not be well suited or designed to target post-discharge patients. ${ }^{8}$ Hospitals directly targeting their community's needs may have a financial incentive to improve social determinants of health if addressing social needs lowers the risk of financial penalties for high readmission rates. The evidence linking lower readmission rates

\footnotetext{
${ }^{1}$ Division of General Internal Medicine, Perelman School of Medicine, University of Pennsylvania, Philadelphia, Pennsylvania.

${ }^{2}$ Leonard Davis Institute of Health Economics, University of Pennsylvania, Philadelphia, Pennsylvania.

${ }^{3}$ Department of Medicine, Cpl. Michael Crescenz VA Medical Center, Philadelphia, Pennsylvania.
} 
related to efforts addressing social determinants of health is lacking.

Community benefit spending reported by nonprofit hospitals and required by the Internal Revenue Service (IRS) provides an opportunity to assess the relationship between hospital-based efforts to address a community's social needs and health care outcomes. Nonprofit hospitals, specifically, are expected to invest in their communities as their taxexempt status depends on recording and reporting the monetary "community benefit" they provide. 9,10 The scope of hospitals' involvement in their communities is broad, ranging from providing health care-related spending for community members (eg, charity care, subsidized care) to making investments that target specific social needs of their local community (eg, community health worker programs to connect patients to social resources, donating funds to local homeless shelters). Therefore, this study examined the association between community benefit spending and nonprofit hospital readmission rates, hypothesizing that hospitals that contribute more resources - financial and material - to their local communities will have lower readmission rates.

\section{Methods}

This study was approved by the institutional review board at the Perelman School of Medicine at the University of Pennsylvania.

\section{Data sources}

A retrospective cohort study of Medicare beneficiaries was conducted between July 1, 2013, through June 30, 2014. Data were obtained from 5 sources: (1) 2013-2014 Section $\mathrm{H}$ and Form 990 IRS tax filings for all nonprofit hospitals, which details community benefit spending for each hospital; (2) 2013-2014 100\% Medicare Provider Analysis and Review files, which include hospital claims for all fee-forservice Medicare beneficiaries; (3) 2013-2014 Medicare Provider of Service Files, which include the characteristics of all Medicare-certified hospitals; (4) 2010-2014 American Community Survey data, which includes zip code-specific sociodemographic data; and (5) the 2013 National Association of County \& City Health Officials' National Profile Study, which describes the jurisdiction of local public health departments.

Tax filings were obtained from Guidestar, which compiles and digitizes tax form data from all nonprofit organizations that file a Form 990 and require supplementary documentation ("Schedules"). Form 990 and Schedule H forms require hospitals to report expenditures that provide community benefit within categories defined by the IRS (Supplementary Tables S1-S4). Each tax filing obtained from Guidestar was matched to a nonprofit hospital listed in the 2013 or 2014 American Hospital Association (AHA) Annual Survey of Hospitals using the name and address of the hospital to create a crosswalk linking the employee identification number in tax filings and the Medicare provider number for nonprofit hospitals in the AHA Annual Survey.

\section{Study sample}

The hospital sample was first limited to private, nonprofit hospitals that filed taxes as singular entities and provided general acute care services to Medicare beneficiaries between July 1, 2013, and June 30, 2014. Hospitals filing jointly with other hospitals were excluded (20.7\% of filings) because their community benefit spending was reported at the health system level (ie, institutions with multiple hospitals or facilities), not the hospital level, and thus spending could not be attributed to the hospital's surrounding community accurately.

For the main analyses, the patient cohort was limited to Medicare beneficiaries who were discharged between July 1, 2013, and June 30, 2014 and resided in a nonprofit hospital's zip code, an estimate of the hospital's local community (this cohort was geographically expanded in sensitivity analyses, which will be described). The nonprofit hospital's surrounding zip codes were chosen as the geographic unit because they represent the most immediate surrounding area likely to benefit from a hospital's community-directed spending. Although spending takes place outside of these zip codes, the size of a zip code was not so large that readmission rates could be influenced by 1 or more neighboring hospitals.

The sample included Medicare beneficiaries who were age 65 years or older, survived their hospitalization, and were discharged to home or another non-acute care setting. Because the main outcome variable was based on Medicare's definition of hospital readmission, Medicare's rules for cohort exclusions ${ }^{11}$ were followed: excluding beneficiaries who were enrolled in Medicare Advantage during the 12 months before discharge (the time period during which comorbidities for risk adjustment were measured) or in the 30 days after hospital discharge (the time period during which readmissions were measured) because claims could not be reliably identified in this cohort; those who were discharged against medical advice or discharged to hospice; and those for whom the primary reason for hospitalization was a psychiatric condition, rehabilitation, or medical cancer treatment.

\section{Main independent variable: community benefit spending}

The main independent variable was a nonprofit hospital's community benefit spending. The 9 categories of community benefit spending specified on the Schedule $\mathrm{H}$ form were grouped into 3 categories: total community benefit spending, and the subcategories of health care-related and communitydirected spending. These 2 subcategories were evaluated specifically because they encompass the majority of community benefit spending. ${ }^{12}$ Previous studies have validated that Form 990 and Schedule $\mathrm{H}$ data align with actual spending. ${ }^{12}$

Health care-related spending included the following categories prespecified on the IRS Schedule $\mathrm{H}$ form: charity care (ie, providing free or discounted services for patients who qualify under a hospital's financial assistance policy), unreimbursed costs incurred by participating in meanstested government programs (eg, Medicaid), and subsidized health services (ie, clinical services provided despite financial loss to the organization not covered by the previous 2 categories). It does not include bad debt or unpaid medical bills. ${ }^{13}$ Community-directed spending included the following: community health improvement services (eg, activities or funds for the direct purposes of improving community 
health, such as community-based anti-smoking campaigns), cash and in-kind contributions for community benefit (eg, monetary contributions to local organizations or health programming), and community-building activities that promote the health of the community the hospital serves (eg, housing, economic development, environmental improvements, leadership development for community members).

These 3 spending measures - total, health care-related, and community-directed - were normalized by calculating the proportion of these spending categories in the hospital's overall expenditure within a tax year to account for differences in total spending across hospitals. The 2 subcategories of health care-related and community-directed spending do not sum to equal total community benefit spending because 2 of the IRS categories, "educational" and "research activities" are not captured in these subcategories but are reported in Schedule $\mathrm{H}$.

\section{Outcome variable: readmission}

The primary outcome was whether a beneficiary had an unplanned readmission for any cause within 30 days of hospital discharge using Medicare's definition. ${ }^{11}$

\section{Covariates}

The research team controlled for the following covariates in adjusted regressions: patient-level covariates including age, race, sex, Medicare/Medicaid dual-enrollment status, and 30 comorbidities defined by Medicare's hospital readmission risk adjustment; hospital characteristics including whether it was a critical access hospital, teaching status, number of beds, urban versus rural location, and Census regions; zip code-level competition based on the HerfindahlHirschman index calculated from the nonprofit hospital's share of acute care beds (accounting for for-profit hospitals) within its zip code; sociodemographics of each beneficiary's zip code including the percent of the zip code's population who were uninsured, unemployed, and had a high school diploma or less; and whether a zip code had a local public health department (city or county), as opposed to only being under the jurisdiction of a state public health department.

\section{Statistical analysis}

To measure the relationship between a hospital's community benefit spending and its readmission rate within its local community, the research team used discharge-level multivariate regression, where the dependent variable was whether or not a discharged patient was readmitted within 30 days and the main independent variable was one of the 3 measures of community benefit spending defined. These 3 spending measures were included separately in the regressions (eg, running one regression for each measure) and each spending measure was included as a set of binary variables representing quintiles of spending (eg, comparing higher quintiles to the lowest) as the relationship between readmissions and spending may not be linear. Absolute differences in the readmission rate and corresponding relative differences were calculated. Adjusted regressions were controlled for the covariates defined. Clustering of patients within hospitals was adjusted for using Huber-White standard errors. $^{14}$
Additional sensitivity analyses were performed to further test the study hypothesis that community-directed spending affects patient outcomes. First, the research team evaluated the association between community-directed spending and readmissions while controlling for health care-related spending. Second, the team excluded $2.6 \%$ of zip codes with $\geq 2$ nonprofit hospitals to concentrate community-directed spending within zip codes with 1 hospital. Third, the team alternatively measured community-directed spending as (a) an absolute dollar amount quantity and (b) per capita community-directed spending calculated based on the population of the zip code. Both are separately included as the main independent variable in the regression models, substituting for the percent of total hospital expenditure used in the primary analysis. Fourth, the team changed the dependent variable to readmissions that are classified as potentially preventable by the Agency for Healthcare Research and Quality, controlling for the same covariates as the primary analysis. ${ }^{11}$ It was expected that potentially preventable readmissions would be more sensitive to patients' social needs and therefore may be more affected by community benefit spending. ${ }^{15}$ Finally, rather than include only patients who lived in the same zip code as the hospital, the team expanded the cohort to patients living within the Hospital Service Area (HSA) for the discharging hospital, ${ }^{16}$ controlling for the covariates defined with modifications for geographically defined covariates (Herfindahl-Hirschman index and sociodemographics) to reflect a larger area. Because community spending was expected to be directed at the hospital's immediate community (ie, its zip code), the team expected that including patients beyond the hospital's zip code would decrease the magnitude of any association between spending and readmissions.

Table 1. Characteristics of Nonprofit Hospitals

\begin{tabular}{lr}
\hline Characteristics & $\begin{array}{r}\text { Nonprofit hospitals* } \\
(N=1405)\end{array}$ \\
\hline Hospital bed size, mean (sd) & $163(189)$ \\
Critical access hospital, n $(\%)$ & $457(32.6)$ \\
Teaching hospital, n (\%) & $219(15.6)$ \\
Urban hospital, n (\%) & $724(51.6)$ \\
Public health department serving & $1351(96.3)$ \\
$\quad$ surrounding zip, n (\%) & \\
Geographic region, n (\%) $\dagger$ & $304(21.7)$ \\
$\quad$ Northeast & $537(38.3)$ \\
Midwest & $375(26.7)$ \\
South & $188(13.4)$ \\
West & $3869(1226)$ \\
Competition within a zip code, & \\
mean (sd) & \\
Community-level variables & $11.5(5.6)$ \\
$\%$ is uninsured, mean (sd) & $7.8(3.9)$ \\
$\%$ is unemployed, mean (sd) & $12.9(7.2)$ \\
$\%$ has a high school diploma or & \\
$\quad$ less, mean (sd) & \\
\hline
\end{tabular}

*Competition was calculated using the Herfindahl-Hirschman index based on the hospital's share of acute care beds (accounting for for-profit hospitals) within its zip code (range 0-10,000).

$\uparrow$ Regions are based on definitions by the United States Census Bureau (https://www.census.gov/geo/reference/gtc/gtc_census_ divreg.html). 
Analyses were performed using SAS 9.3 (SAS Institute Inc., Cary, NC) and Stata software, version 14.1 (StataCorp LLC, College Station, TX). The 95\% confidence intervals (CIs) around reported estimates reflect 0.025 in each tail, or $P$ values no higher than 0.05 .

\section{Results}

The 1405 nonprofit hospitals that met eligibility criteria were included (Table 1). These hospitals had an average of 163 beds (standard deviation [SD] 189 beds), were predominantly urban, non-critical access, and nonteaching hospitals, with the majority residing within a local public health department's jurisdiction. The average HerfindahlHirschman index was 0.39 (SD 0.12), reflecting highly concentrated markets (ie, low levels of competition). ${ }^{17}$ The

Table 2. Characteristics of Discharged Patients

\begin{tabular}{|c|c|}
\hline Characteristics & $\begin{array}{l}\text { Discharges from nonprofit } \\
\text { hospital who reside in the } \\
\text { hospital's zip code } \\
\quad(N=341,913)\end{array}$ \\
\hline Readmissions, No. (\%) & $53,308(15.6)$ \\
\hline Age, mean (sd) & $80.4(8.4)$ \\
\hline \multicolumn{2}{|l|}{ Race, No. (\%) } \\
\hline White & $304,805(89.2)$ \\
\hline Black & $25,196(7.4)$ \\
\hline Hispanic & $359(1.1)$ \\
\hline Other & $8153(2.4)$ \\
\hline Female, No. $(\%)$ & $206,434(60.4)$ \\
\hline Dual eligibles, No. (\%) & $67,041(19.6)$ \\
\hline \multicolumn{2}{|l|}{ Comorbidities, No. (\%) } \\
\hline Arrhythmia & $79,208(23.2)$ \\
\hline Arthritis & $18,414(5.4)$ \\
\hline Cancer, metastatic & $10,289(3.0)$ \\
\hline Cancer, other & $23,926(7.0)$ \\
\hline Cancer, severe & $17,062(5.0)$ \\
\hline Cardiorespiratory & $19,931(5.8)$ \\
\hline Congestive heart failure & $41,458(12.1)$ \\
\hline $\begin{array}{l}\text { Chronic obstructive } \\
\text { pulmonary disease }\end{array}$ & $102,440(30.0)$ \\
\hline Coagulopathy & $18,017(5.3)$ \\
\hline $\begin{array}{l}\text { Coronary artery disease, } \\
\text { cardiovascular disease }\end{array}$ & $194,489(56.9)$ \\
\hline Dependent on respirator & $988(0.3)$ \\
\hline Diabetes & $122,710(35.9)$ \\
\hline Hematologic disorder & $3716(1.1)$ \\
\hline Hip fracture & $8528(2.5)$ \\
\hline History of infection & $4128(1.2)$ \\
\hline Infections (other) & $90,874(26.6)$ \\
\hline Iron deficiency & $152,761(44.7)$ \\
\hline Liver disease & $5204(1.5)$ \\
\hline Lung disorder & $13,461(3.9)$ \\
\hline Malnutrition & $31,897(9.3)$ \\
\hline Metabolic disorder & $95,873(28.0)$ \\
\hline Motor dysfunction & $17,350(5.1)$ \\
\hline Pancreatic disease & $6804(2.0)$ \\
\hline Psychologic disorder & $101,174(29.6)$ \\
\hline Receiving dialysis & $5707(1.7)$ \\
\hline Renal failure & $73,662(21.5)$ \\
\hline Seizure & $14,466(4.2)$ \\
\hline Septicemia & 22,567 (6.6) \\
\hline Transplant & $1247(0.4)$ \\
\hline Ulcers & $21,171(6.2)$ \\
\hline
\end{tabular}

percentages of uninsured, unemployed, and those with a high school diploma or less are shown in Table 1. These hospitals discharged 341,913 Medicare beneficiaries over the study period, who were predominantly elderly, white, female, and had a wide array of comorbidities (Table 2).

Among these hospitals, total community benefit spending averaged $8 \%$ of total hospital expenditures, health carerelated spending averaged $<7 \%$ of total spending, and community-directed spending averaged $<1 \%$ of total spending (Table 3). In dollar terms, these average expenditures equaled \$14.8 million, \$11.1 million, and \$1.0 million, respectively. There was variability in community benefit spending across hospitals. Quintile ranges of spending for total community benefit, health care-related spending, and community-directed spending are shown in Table 3.

Total community benefit spending and health care-related spending were not associated with readmission rates in adjusted analyses (Figure 1). In contrast, hospitals with higher levels of community-directed spending had lower readmission rates across all 5 categories of community-directed spending in adjusted analyses. Compared with patients discharged from hospitals with the lowest levels of communitydirected spending (the first quintile), patients discharged from hospitals in the second quintile had a readmission rate that was 0.82 percentage points lower $(95 \% \mathrm{CI}-1.48$ to $-0.16 ; P=0.01), 1.02$ percentage points lower $(95 \% \mathrm{CI}$ -1.67 to $-0.36 ; P<0.01)$ in the third quintile, 1.03 percentage points lower $(95 \% \mathrm{CI}-1.73$ to $-0.34 ; P<0.01)$ in the fourth quintile, and 1.21 percentage points lower $(95 \% \mathrm{CI}$ -1.85 to $-0.56 ; P<0.001)$ in the fifth quintile.

Table 3. Community Benefit Spending BY NonPROFIT Hospitals

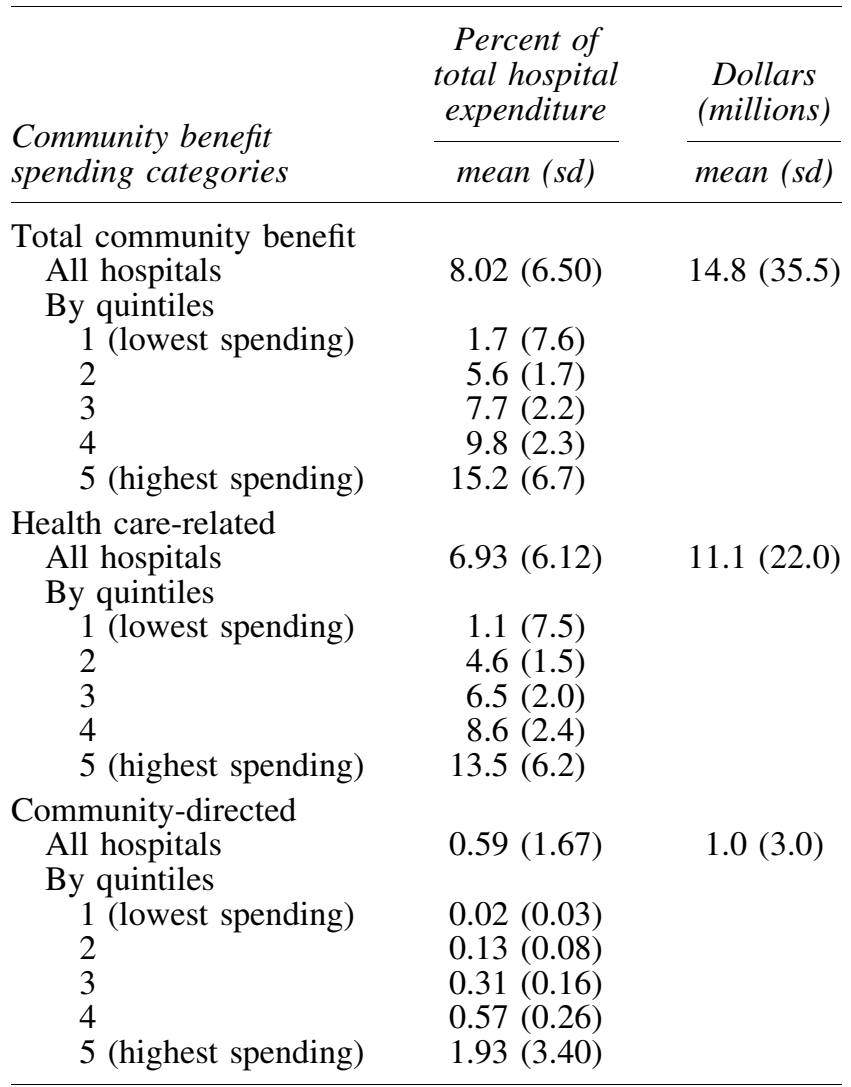



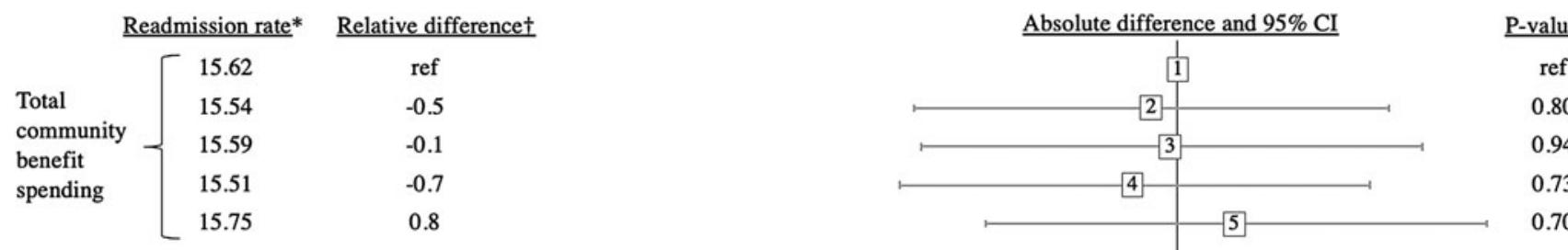

Health care-
related
spending $\begin{cases}15.61 & \text { ref } \\ 15.51 & -0.6 \\ 15.57 & -0.2 \\ 15.64 & 0.2 \\ 15.64 & 0.2\end{cases}$

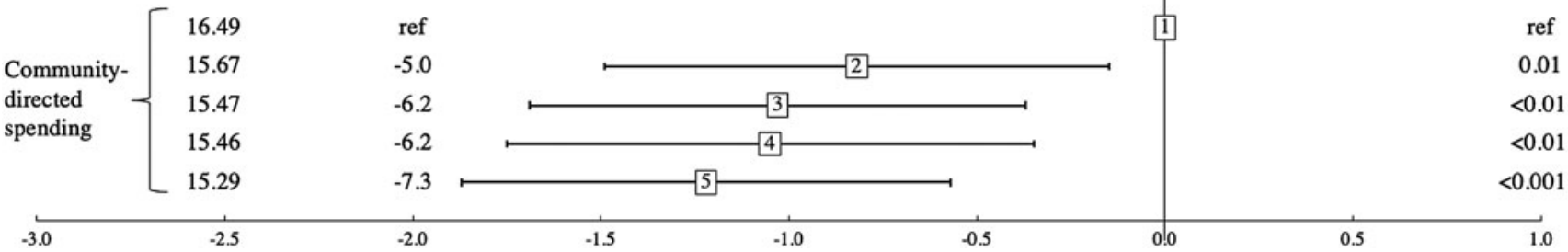

FIG. 1. Association between quintiles of community benefit spending categories and all-cause readmission rates at nonprofit hospitals. *Adjusted for community sociodemographics (ZIP codes within the jurisdiction of a local public health department and percent of ZIP code who were uninsured, unemployed, and had high school degree or less), hospital characteristics (being a critical access hospital, teaching hospital, hospital bed size, urban versus rural location, and level of market competition based on the Herfindahl-Hirschman index calculated from the hospital's share of the market for acute care services within each ZIP code, and the geographic region), and patient characteristics (age, race, sex, and 31 comorbidities). †Changes in readmission rate are compared to the reference group of the lowest quartile (e.g., the quartile with the lowest spending).

When including community-directed spending and health care-related spending in the same model (Supplementary Table S1) and excluding the $2.6 \%$ of discharges occurring within zip codes with more than 1 hospital (Supplementary Table S2), study findings were largely unchanged. When changing how community-directed spending was measured (ie, absolute dollar amounts or per capita spending), the findings remain consistent: higher levels of communitydirected spending were associated with lower readmission rates than the lowest level of spending (Supplementary Table S3). When focusing on the subgroup of preventable readmissions, neither total community-directed nor health care-related spending was associated with rates of preventable readmissions, although higher rates of communitydirected spending were associated with significantly lower readmission rates for the top 3 quintiles of spending (Supplementary Table $\mathrm{S} 4$ ). The relative difference in rates of potentially preventable readmissions between these 3 highest quintiles and the hospitals with the lowest levels of community-directed spending was more than 1.5-fold higher than the difference in the rate for all zip code-level readmissions. When expanding the patient cohort to include patients living in the hospital's HSA (rather than in the hospital's zip code), higher quintiles of total community and health care-related spending again were not associated with meaningful differences in readmission rates (Supplementary Table S4). Community-directed spending resulted in significantly lower readmission rates for higher quintiles of spending, but the magnitude of these differences was smaller than readmission rates for patients living in the same zip code as the hospital.

\section{Discussion}

In a large national sample of patients who were enrolled in Medicare and lived in the same zip code as the hospital where they received care, the associations between readmission rates and total community benefit spending and the health care-related subset were not statistically significant. Lower readmission rates were observed at hospitals with higher levels of community-directed spending. The differences in readmission rates between upper levels of community-directed spending and the lowest level of spending were larger among potentially preventable readmissions, which are more susceptible to social needs, ${ }^{15,18}$ and smaller when including patients living farther from the hospital.

To provide context for the association observed between readmissions and community-directed spending, readmission rates for conditions targeted by the Hospital Readmissions Reduction Program decreased nationally by 3.7 percentage points (from $21.5 \%$ to $17.8 \%$ ) over an 8 -year period between 2007 and $2015 .^{19}$ At the same time, readmission rates for non-targeted conditions declined by 2.2 percentage points (from $15.3 \%$ to $13.1 \%$ ). ${ }^{19,20}$ The variation in readmission rates reported in the present study is nearly half as much - ranging from 0.82 to 1.21 percentage points across hospitals. This variation, however, was associated 
with relatively low levels of spending on community health needs.

This work has several limitations. Most importantly, this is an observational study based on cross-sectional data and the findings cannot be interpreted as causal. However, yearto-year community benefit spending has not changed significantly since $2012^{21}$ and the magnitude of associations documented varied across different specifications (eg, changes in definition of community, a greater magnitude effect on preventable readmissions) as would be expected if communitydirect spending affected readmission rates. Second, present study findings are not generalizable beyond nonprofit hospitals or non-Medicare readmissions. Third, the research team cannot comment on the specific types of communitydirected spending that were associated with readmission rates because hospitals did not consistently describe what they specifically invested beyond the categories listed. Fourth, community-directed spending may be a proxy for a mission-driven hospital focused on reducing readmissions for the socially vulnerable. Although, if mission-driven hospitals are addressing social barriers to care, this may reinforce the study hypothesis that social investments toward community members' needs may lead to reductions in readmissions. Fifth, alternative explanations for the study findings may exist. Hospitals with higher readmission rates incurred more penalties under Medicare's Hospital Readmissions Reduction Program, resulting in limited financial capacity to invest in community-directed spending. As a result, present study findings may be related to lower performing hospitals spending less on community needs, not necessarily better performing hospitals spending more on community needs. Sixth, these results may be influenced by selection bias, whereby patients who are healthier are going to hospitals that are more charitable. Although this study controls for a number of observable patient characteristics, the possibility of residual biases remains. Seventh, the zip code may be an inaccurate representation of a hospital's community as hospitals may define communities with larger or smaller geographic areas depending on factors such as geography and market competition. This study uses the hospital's zip code as an approximate estimate for a hospital's immediate community, balancing the need to have a wide enough geographic region while not being so broad that a geographic area's readmission rate could be influenced by 1 or more neighboring hospitals. Further, the research team is unable to capture which zip received the most community benefit spending because of the limited information provided on the IRS Section H forms. Finally, hospitals, local community organizations, or public health departments may invest in their local communities in ways that are not captured in the data set. Although a number of variables are controlled for, such as the presence of a local public health department, these unmeasured activities may alter the estimates.

Nonetheless, these findings suggest that nonprofit hospitals investing in their communities may be associated with the health outcomes of individuals living in those communities. Ultimately, understanding the influence of social and economic factors on health outcomes will become even more important as the health sector becomes increasingly accountable for the health of their patients and their communities. Further efforts are needed to measure, understand, and validate the relationship between hospital-based efforts to address social determinants of health and their broader impact on health outcomes that increasingly matter under value-based payment reforms. Aligning disease-based population health efforts with social interventions may be a critical strategy to achieve desired health outcomes.

\section{Author Disclosure Statement}

Dr. Werner is a consultant for CarePort Health. The other authors declare that there are no conflicts of interest. This work was supported by a grant from the National Institute on Aging (K24-AG047908) to Dr. Werner. Dr. Chaiyachati received funds for training support from the Robert Wood Johnson Foundation Clinical Scholars Program and the VA Office of Academic Affairs during the time of the study. The funding agency played no role in the design and conduct of the study; collection, management, analysis, and interpretation of the data; preparation, review, or approval of the manuscript; and decision to submit the manuscript for publication.

\section{Supplementary Material}

Supplementary Table S1

Supplementary Table S2

Supplementary Table S3

Supplementary Table S4

\section{References}

1. Gu Q, Koenig L, Faerberg J, Steinberg CR, Vaz C, Wheatley MP. The Medicare hospital readmissions reduction program: potential unintended consequences for hospitals serving vulnerable populations. Health Serv Res 2014;49:818-837.

2. The National Academy of Medicine. Accounting for social risk factors in Medicare payment: identifying social risk factors. Washington, DC: National Academies Press, 2016.

3. Joynt KE, Orav EJ, Jha AK. Thirty-day readmission rates for Medicare beneficiaries by race and site of care. JAMA 2011;305:675-681.

4. Chaiyachati KH, Qi M, Werner RM. Changes to racial disparities in readmission rates after Medicare's hospital readmissions reduction program within safety-net and nonsafety-net hospitals. JAMA Netw Open 2018;1:e184154.

5. Joynt KE, Jha AK. Characteristics of hospitals receiving penalties under the hospital readmissions reduction program. JAMA 2013;309:342-343.

6. Dzau VJ, McClellan MB, McGinnis J, et al. Vital directions for health and health care: Priorities from a national academy of medicine initiative. JAMA 2017;317:1461-1470.

7. Chetty R, Stepner M, Abraham S, et al. The association between income and life expectancy in the United States, 2001-2014. JAMA 2016;315:1750-1766.

8. Taylor LA, Tan AX, Coyle CE, et al. Leveraging the social determinants of health: what works? PLoS One 2016;11: e0160217.

9. Nikpay SS, Ayanian JZ. Hospital charity care-effects of new community-benefit requirements. N Engl J Med 2015; 373:1687-1690.

10. Rosenbaum S, Margulies R. Tax-exempt hospitals and the Patient Protection and Affordable Care Act: implications for public health policy and practice. Public Health Rep 2011;126:283-286. 
11. National Quality Measures C. Unplanned readmission: hospital-wide all-cause, unplanned readmission rate (HWR). 2015. https://cmit.cms.gov/CMIT_public/ViewMeasure? MeasureId=2593 Accessed April 11, 2018.

12. Young GJ, Chou CH, Alexander J, Lee SY, Raver E. Provision of community benefits by tax-exempt U.S. hospitals. N Engl J Med 2013;368:1519-1527.

13. Health Affairs. Health Policy Brief: Nonprofit Hospitals' Community Benefit Requirements. February 25, 2016. https:// www.healthaffairs.org/do/10.1377/hpb20160225.954803/abs/ Accessed April 11, 2018.

14. White H. A heteroskedasticity-consistent covariance matrix estimator and a direct test for heteroskedasticity. Econometrica 1980;48:817-830.

15. Vest JR, Gamm LD, Oxford BA, Gonzalez MI, Slawson KM. Determinants of preventable readmissions in the United States: a systematic review. Implement Sci 2010;5: 88.

16. The Center for the Evaluative Clinical Sciences DMS. The Dartmouth Atlas of Health Care. www.dartmouthatlas.org/ data/region/ Accessed February 2, 2017.

17. U.S. Department of Justice and the Federal Trade Commission. Horizontal Merger Guidelines. 2010. www .ftc.gov/system/files/documents/public_statements/804291/ 100819hmg.pdf Accessed April 11, 2018.
18. Hu J, Gonsahn MD, Nerenz DR. Socioeconomic status and readmissions: evidence from an urban teaching hospital. Health Aff (Millwood) 2014;33:778-785.

19. Zuckerman RB, Sheingold SH, Orav EJ, Ruhter J, Epstein AM. Readmissions, observation, and the hospital readmissions reduction program. N Engl J Med 2016;374:15431551.

20. Demiralp B, He F, Koenig L. Further evidence on the system-wide effects of the hospital readmissions reduction program. Health Serv Res 2018;53:1478-1497.

21. Chaiyachati KH, Qi M, Werner RM. Non-profit hospital community benefit spending based on local sociodemographics. J Health Care Poor Underserved 2018;29: 1259-1268.

Address correspondence to: Krisda H. Chaiyachati, MD, MPH, MSHP Division of General Internal Medicine Perelman School of Medicine University of Pennsylvania 423 Guardian Drive, 1313 Blockley Hall Philadelphia, PA 19104

E-mail: kchai@pennmedicine.upenn.edu 